\title{
Identification of Piecewise Affine Systems Using Sum-of-Norms Regularization
}

\author{
Henrik Ohlsson and Lennart Ljung
}

\section{Linköping University Post Print}

\section{Tweet}

N.B.: When citing this work, cite the original article.

Original Publication:

Henrik Ohlsson and Lennart Ljung, Identification of Piecewise Affine Systems Using Sumof-Norms Regularization, 2011, Proceedings of the 18th IFAC World Congress, 2011, 66406645 .

http://dx.doi.org/10.3182/20110828-6-IT-1002.00611

ISBN: 978-3-902661-93-7

The 18th IFAC World Congress, August 28-September 2. Milan, Italy, 2011

Copyright: IFAC

www.ifac-papersonline.net/

Postprint available at: Linköping University Electronic Press http://urn.kb.se/resolve?urn=urn:nbn:se:liu:diva-60984 


\title{
Identification of Piecewise Affine Systems Using Sum-of-Norms Regularization *
}

\author{
Henrik Ohlsson, Lennart Ljung \\ Department of Electrical Engineering, Linköping University, \\ SE-581 83 Linköping, Sweden
}

\begin{abstract}
Piecewise affine systems serve as an important approximation of nonlinear systems. The identification of piecewise affine systems is here tackled by overparametrizing and assigning a regressor-parameter to each of the observations. Regressor parameters are then forced to be the same if that not causes a major increase in the fit term. The formulation takes the shape of a least-squares problem with sum-of-norms regularization over regressor parameter differences, a generalization of $\ell_{1}$-regularization. The regularization constant is used to trade off fit and the number of partitions.
\end{abstract}

\section{INTRODUCTION}

Hybrid systems is a class of systems having both continuous and discrete dynamics. The continuous dynamics are often ruled by physical principles and the discrete due to discrete decisions or logic devices. But hybrid systems have also proven to be handy approximations of nonlinear continuous systems.

A type of hybrid systems is systems which can be described by a piecewise affine function, denoted piecewise affine systems. A piecewise affine (PWA) function $f: R^{n_{x}} \rightarrow$ $R^{n_{y}}$ can be written on the form

$$
f(x)= \begin{cases}\theta_{1}^{T}\left[\begin{array}{l}
x \\
1
\end{array}\right], & \text { if } x \in \mathcal{H}_{1}, \\
\vdots & \\
\theta_{s}^{T}\left[\begin{array}{l}
x \\
1
\end{array}\right], & \text { if } x \in \mathcal{H}_{s} .\end{cases}
$$

$s$ is the number of partitions. The partitions are often restricted to be polyhedral. Measurements, or observations, are noisy versions of $f(x)$ according to

$$
y=f(x)+e, \quad E[e]=0, E\left[e e^{T}\right]=\Gamma .
$$

A subclass of PWA functions is piecewise ARX (PWARX). For a PWARX, $x$ is composed of past system inputs $u$ and outputs $y$.

\subsection{Problem Formulation}

Given the observations $\left\{\left(y_{k}, x_{k}\right)\right\}_{k=1}^{N}, y \in R, x \in R^{x}$, estimate a piecewise affine function of the form (1). The number of partitions, $s$, is a priori unknown. Estimation of the shape of the partitions is not treated in this contribution but can be handled by e.g., applying a classification algorithm to the output of the proposed algorithm (see e.g., Bemporad et al. [2005]).

\footnotetext{
* Partially supported by the Swedish foundation for strategic research in the center MOVIII and by the Swedish Research Council in the Linnaeus center CADICS.
}

\subsection{Background}

It is clear that if the partitions, i.e., $\mathcal{H}_{i}, i=1, \ldots, s$, are known, it is easy to find the regressor parameters of the subsystems. PWA system identification approaches can therefore be classified into groups according to how they find the partitions. Five techniques stand out:

- The parameters giving the partitions and the subsystem models are estimated simultaneously.

- Simple partitions and subsystem models are estimate simultaneously and repeatedly. See e.g., Roll et al. [2004].

- The partitions and submodels are iteratively estimated, alternating between estimating partitions and submodels. See e.g., Bemporad et al. [2003].

- The partitions are first estimated and then the submodels.

- The submodels are estimated and then the partitions (see e.g., Vidal et al. [2003], Bemporad et al. [2005]).

The proposed method belongs to the last category. The underlying idea of methods of the last item is to simultaneously cluster and fit an affine model to the data of each cluster. It is essential that the clustering and regression are done simultaneously (or possibly alternating between the two) since the distance measure used in the clustering can not only be based on the distance between regressors. It must also depend on how well the measured output fit to the estimated submodels.

In this contribution we pose the identification of piecewise affine systems as a sum-of-norms regularized least squares problem. The regularization constant is used to trade off fit and the number of partitions i.e., s, and could preferably be found using cross validation. The proposed formulation takes the form of a convex optimization problem, so the global solution can be computed efficiently. Relevant previous contributions using the sum-of-norms regularization are Kim et al. [2009], Ohlsson et al. [2010c,a,b]. See also Ozay et al. [2008]. 


\section{PROPOSED METHOD}

\subsection{Informal Preview}

(1) We are given a data set $Z^{N}=\left\{y_{k}, x_{k}, k=1, \ldots, N\right\}$. In a first round we associate each measurement $k$ with a parameter vector $\theta_{k} \in R^{n_{x}+1}$.

(2) Then we cluster the $x_{k}$ into $s$ subsets $H_{r}=\left\{x_{k} \mid k \in\right.$ $\left.K_{r}\right\}, r=1, \ldots, s$, that are suitable to associate with the same vector $\bar{\theta}_{r}$.

(3) This is done by checking which parameter vectors can be merged $\theta_{k}=\theta_{j}=\bar{\theta}_{r}$ for $k, j \in K_{r}$ at the smallest cost of fit for the output observations

$$
\left\|\Gamma^{-1 / 2}\left(y_{k}-\bar{\theta}_{r}^{T}\left[\begin{array}{c}
x_{k} \\
1
\end{array}\right]\right)\right\|_{2}^{2} .
$$

(4) This gives a function $r(k)$ that assigns the observation $k$ to a subset $r, s$ parameter vectors $\bar{\theta}_{r}$ and $s$ point sets $H_{r}$.

(5) The point sets $H_{r}$ can now the used to partition the $x$ space into $s$ partitions $\mathcal{H}_{r}$. This is a standard pattern recognition/classification problem that can be solved by several established technique (e.g., support vector machines [Vapnik, 1995]) and will not be discussed here. See also Bemporad et al. [2005] for a discusses of this problem for a PWA system identification setting.

\subsection{Clustering Algorithm}

We solve step (3) by the following technique: Let

$$
K\left(x_{k}, x_{j}\right): R^{n_{x}} \times R^{n_{x}} \rightarrow R
$$

be a kernel. We will give some examples of suitable choices of $K$ shortly.

Given a data set $Z^{N}$, minimize

$\sum_{k=1}^{N}\left\|\Gamma^{-1 / 2}\left(y_{k}-\theta_{k}^{T}\left[\begin{array}{c}x_{k} \\ 1\end{array}\right]\right)\right\|_{2}^{2}+\lambda \sum_{k, j=1}^{N} K\left(x_{k}, x_{j}\right)\left\|\theta_{k}-\theta_{j}\right\|_{p}$

with respect to $\theta_{k}, k=1, \ldots, N$, where $\Gamma$ is defined in (2). We define:

- $s$ as the number of distinct $\theta$-values in $\left\{\theta_{k}, k=\right.$ $1, \ldots, N\}\left(\theta_{k}, k=1, \ldots, N\right.$ minimizing $\left.(5)\right)$.

- $\bar{\theta}_{r}, r=1, \ldots, s$ to be the $s$ distinct $\theta$-values of $\left\{\theta_{k}, k=1, \ldots, N\right\}$.

- $H_{r}, r=1, \ldots, s$ as $H_{r} \triangleq\left\{x_{k} \mid \theta_{k}=\bar{\theta}_{r}\right\}$.

- $r(k)$ as the function

$$
r(k) \triangleq r \mid k \in H_{r} .
$$

The first term of (5),

$$
\sum_{k=1}^{N}\left\|\Gamma^{-1 / 2}\left(y_{k}-\theta_{k}^{T}\left[\begin{array}{c}
x_{k} \\
1
\end{array}\right]\right)\right\|_{2}^{2}
$$

measures the fit to observations. The second term

$$
\sum_{k=1}^{N} \sum_{j=1}^{N} K\left(x_{k}, x_{j}\right)\left\|\theta_{k}-\theta_{j}\right\|_{p}
$$

is a regularization term. Since the number of parameters in (5) equals the number of observations, the regularization is necessary to prevent overfitting to the noisy observations. Using (8) we prevent overfitting by penalizing the number of distinct $\theta$-values, essentially $s$, used in in (5).
Remark 1. Undesirable, also the cardinalities of $H_{r}, r=$ $1, \ldots, s$, plays a role in the regularization (8). Our experience is that this effect is minor and that $\lambda$ controls the trade-off between fit and the number of partitions $s$.

When the regularization norm is taken to be the $\ell_{1}$ norm, i.e., $\|z\|_{1}=\sum_{i=1}^{n_{z}}\left|z_{i}\right|$, the regularization in (5) is a standard $\ell_{1}$ regularization of the least-squares criterion. Such regularization has been very popular recently, e.g., in the much used Lasso method, [Tibsharani, 1996] or compressed sensing [Donoho, 2006, Candès et al., 2006].

There are two key reasons why the criterion (5) is attractive:

- It is a convex optimization problem, so the global solution can be computed efficiently.

- The sum-of-norms-regularization (a generalization of the $\ell_{1}$-regularization) will cause $\theta_{k}$ to be identical to $\theta_{r}$, if that not causes a major increase in the fit term (7). In this case, this implies that many of the regularized variables come out as exactly zero. $\lambda$ is a design parameter which regulates the number of partitions found.

- It is easy to include constraints without destroying convexity.

The kernel can be used to stress that $\theta$ :s associated with closed-by $x$ :s are more probable to have identical $\theta$-values. It can be seen as a prior for the clustering. We will use the following kernel in our examples:

$$
K\left(x_{k}, x_{r}\right) \triangleq \begin{cases}1 & \text { if } x_{r} \text { is one of the } 9 \text { closest neighbors } \\ 0 & \text { of } x_{k} \text { among all the observations, }\end{cases}
$$

We should comment on the difference between using a $\ell_{1}$ regularization and some other type of sum-of-norms regularization, such as sum-of-Euclidean norms. With $\ell_{1}$ regularization, we obtain an estimate of the regularization variable having many of its components equal to zero. When we use sum-of-norms regularization, the whole estimated regularization variable vector often becomes zero; but when it is nonzero, typically all its components are nonzero. In a statistical linear regression framework, sumof-norms regularization is called Group-Lasso [Yuan and Lin, 2006], since it results in estimates in which many groups of variables are zero.

\subsection{Iterative Refinement}

To (possibly) get even more zeros in the estimate of the regularized variables, with no or small increase in the fitting term, iterative re-weighting can be used [Candès et al., 2008]. We modify the regularization term in (5) and consider

$$
\begin{aligned}
& \sum_{k=1}^{N}\left\|\Gamma^{-1 / 2}\left(y_{k}-\theta_{k}^{T}\left[\begin{array}{c}
x_{k} \\
1
\end{array}\right]\right)\right\|_{2}^{2} \\
& \quad+\lambda \sum_{k=1}^{N} \sum_{j=1}^{N} \alpha(k, j) K\left(x_{k}, x_{j}\right)\left\|\theta_{k}-\theta_{j}\right\|_{p}
\end{aligned}
$$

where $\alpha(1,1), \ldots, \alpha(N, N)$ are positive weights used to vary the regularization over indices $j$ and $k$. Iterative 
refinement proceeds as follows. We start with all weights equal to one i.e., $\alpha^{(0)}(k, j)=1$. Then for $i=0,1, \ldots$ we carry out the following iteration until convergence (which is typically in just a few steps).

(1) Find the $\theta$ estimates.

Compute the optimal $\theta_{k}^{(i)}$ using (10) with the weighted regularization using weights $\alpha^{(i)}$.

(2) Update the weights.

For $j=1, \ldots, N$, set $\alpha^{(i+1)}(k, j)=1 /\left(\epsilon+\left\|\theta_{k}-\theta_{j}\right\|_{p}\right)$.

Here $\epsilon$ is a positive parameter that sets the maximum weight that can occur.

One final step is also useful. From our final estimate of $\bar{\theta}$, we simply define the mapping $r(k)$ (see (6)) from the last iteration. Then carry out a constrained least squares optimization over $\bar{\theta}_{r}$

$$
\min _{\bar{\theta}_{r}, r=1, \ldots, s} \sum_{k=1}^{N}\left\|\Gamma^{-1 / 2}\left(y_{k}-\bar{\theta}_{r(k)}^{T}\left[\begin{array}{c}
x_{k} \\
1
\end{array}\right]\right)\right\|_{2}^{2} .
$$

The algorithm is summarized in Algorithm 1.

Algorithm 1. PWA System Identification Using Sum-ofNorms Regularization (PWASON)

Given $\left\{\left(y_{t}, x_{t}\right)\right\}_{t=1}^{N}$. Let $\epsilon$ be a positive parameter, set $\alpha^{(0)}(k, j)=1$ for $k, j=1, \ldots, N$ and $i=0$. Then, for a chosen kernel $K, p$ and regularization parameter $\lambda$ :

(1) Compute the optimal $\theta_{k}^{(i)}$ using (10) with $\alpha=\alpha^{(i)}$.

(2) Set $\alpha^{(i+1)}(k, j)=1 /\left(\epsilon+\left\|\theta_{k}-\theta_{j}\right\|_{p}\right)$.

(3) If convergence, go to the next step, otherwise set $i=i+1$ and return to (1).

(4) Compute a final estimate of $\bar{\theta}_{r}$ using (11).

\subsection{Solution Algorithms and Software}

Many standard methods of convex optimization can be used to solve the problem (5). Systems such as CVX [Grant and Boyd, 2010, 2008] or YALMIP [Löfberg, 2004] can readily handle the sum-of-norms regularization, by converting the problem to a cone problem and calling a standard interior-point method. For the special case when the $\ell_{1}$ norm is used as the regularization norm, more efficient special purpose algorithms and software can be used, such as 11_ls [Kim et al., 2007]. Recently many authors have developed fast first order methods for solving $\ell_{1}$ regularized problems, and these methods can be extended to handle the sum-of-norms regularization used here; see, for example, Roll [2008§2.2].

\section{NUMERICAL ILLUSTRATIONS}

\section{Example 3.1. A One Dimensional Example}

Consider the one-dimensional PWARX system (introduced in Ferrari-Trecate et al. [2003])

$$
y_{k}= \begin{cases}u_{k-1}+2+e_{k}, & -4 \leq u_{k-1} \leq-1, \\ -u_{k-1}+e_{k}, & -1<u_{k-1}<2 \\ u_{k-1}+2+e_{k}, & 2 \leq u_{k-1} \leq 4\end{cases}
$$

Generate $\left\{u_{k}\right\}_{k=1}^{50}$ by sample a uniform distribution $U(-4,4)$. Let $e_{k} \sim N(0,0.05)$. Figure 1 shows the dataset $\left\{\left(y_{k}, u_{k}\right)\right\}_{k=1}^{50}$. Let the kernel $K$ be defined by (9), set

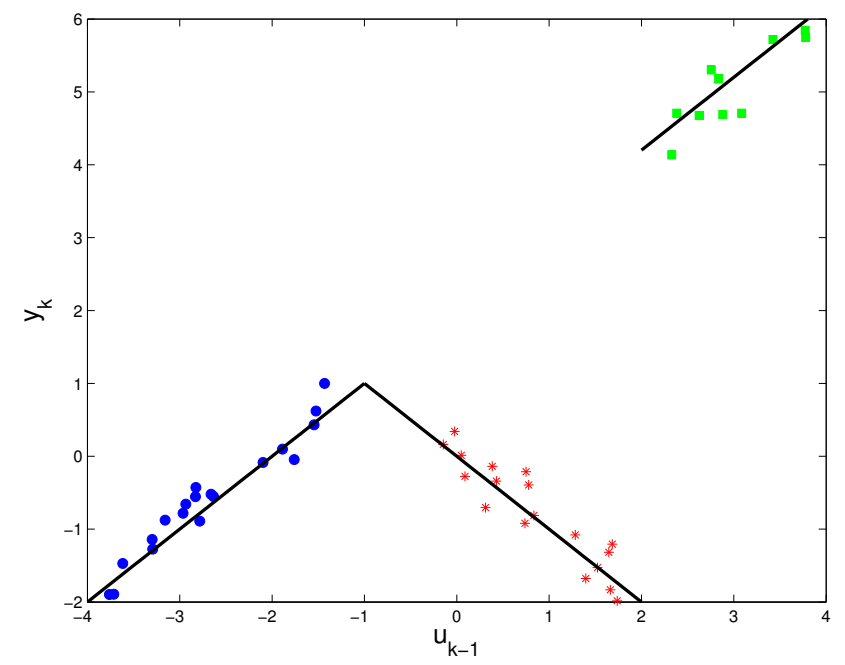

Fig. 1. Data used in Example 3.1. Solid line shows the true PWA function of the PWARX system.

$x_{k}=u_{k-1}, \Gamma=1$ and chose $p=2 . \lambda=2$ then produces the result shown in Figure 2. The obtained $\bar{\theta}$-values were:

$$
\left[\begin{array}{c}
-1.0 \\
0.1
\end{array}\right],\left[\begin{array}{l}
1.0 \\
2.2
\end{array}\right],\left[\begin{array}{l}
1.0 \\
2.1
\end{array}\right] \text {. }
$$

The results compare well with the result reported in FerrariTrecate et al. [2003].
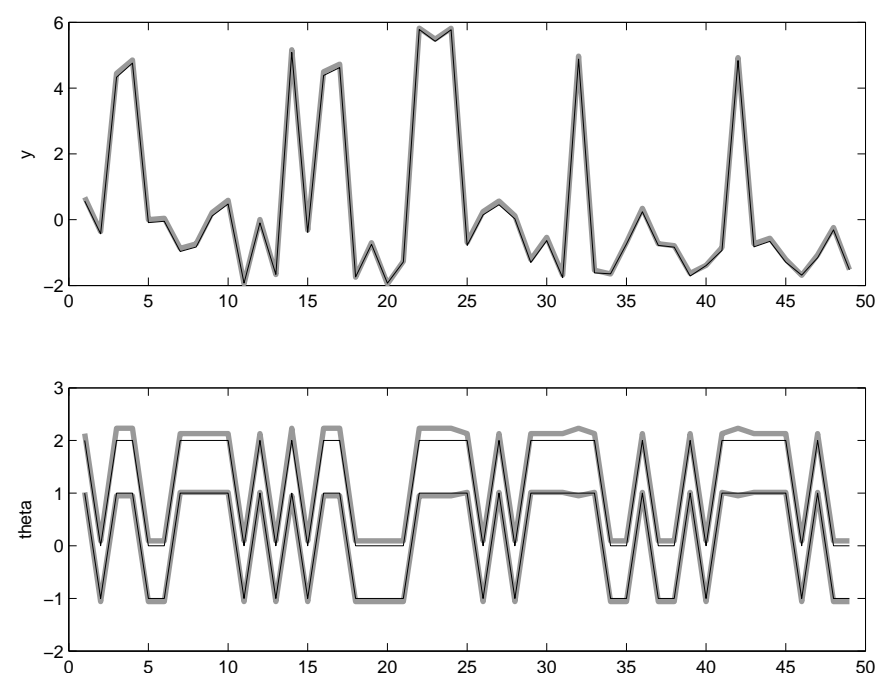

Fig. 2. Top plot, true (thin black line) and estimated (thick gray line) $y$ (underneath the black line so hardly visible) for $k=1, \ldots, 50$. Bottom plot, true (thin black line) and estimated (thick gray line) $\theta$ for $k=1, \ldots, 50$.

Example 3.2. A Multi-Dimensional Example

Consider the multi-dimensional PWARX system (introduced in Bemporad et al. [2003], see also Nakada et al. [2005], Bemporad et al. [2005])

$$
y_{k}=\left\{\begin{aligned}
&-0.4 y_{k-1}+ u_{k-1}+1.5+e_{k}, \text { if } \\
& 4 y_{k-1}-u_{k-1}+10<0 \\
& 0.5 y_{k-1}- u_{k-1}-0.5+e_{k}, \text { if } \\
& 4 y_{k-1}-u_{k-1}+10 \geq 0 \text { and } \\
& 5 y_{k-1}+u_{k-1}-6<0 \\
&-0.3 y_{k-1}+ 0.5 u_{k-1}-1.7+e_{k}, \text { if } \\
& 5 y_{k-1}+u_{k-1}-6 \geq 0 .
\end{aligned}\right.
$$


Generate $\left\{u_{k}\right\}_{k=1}^{200}$ by sample a uniform distribution $U(-4,4)$ and let $e_{k} \sim U(-0.2,0.2)$. Figure 3, top plot, shows the dataset $\left\{\left(y_{k}, u_{k}\right)\right\}_{k=1}^{200}$. Define the kernel $K$ as in (9), set $x_{\underline{k}}=\left[y_{k-1} u_{k-1}\right]^{T}, \Gamma=1, p=2$ and $\lambda=1$. The obtained $\theta$-values were:

$$
\left[\begin{array}{c}
-0.40 \\
1 \\
1.50
\end{array}\right],\left[\begin{array}{c}
0.50 \\
-1 \\
-0.50
\end{array}\right],\left[\begin{array}{c}
0.57 \\
-1 \\
-0.50
\end{array}\right],\left[\begin{array}{c}
-0.30 \\
0.50 \\
-1.7
\end{array}\right],\left[\begin{array}{c}
-1.60 \\
1.92 \\
-4.7
\end{array}\right]
$$

Most of the observations obtained a $\theta$ equal to one of the four first $\bar{\theta}$-estimates in (15). Three observations got a $\theta$-estimate equal to the fifth estimate. Increasing $\lambda$ $(\lambda=1.2)$ causes the third $\theta$-estimate to disappear and the observations previously associated with it to change to the second $\theta$-estimate. This estimate is visualized in the bottom of Figure 3, Figure 4, 5, 6 and 7. $s$ is then 4 . Setting $\lambda=1.5$ makes $s=3$ and by that, all observations were correctly assigned to their partition.
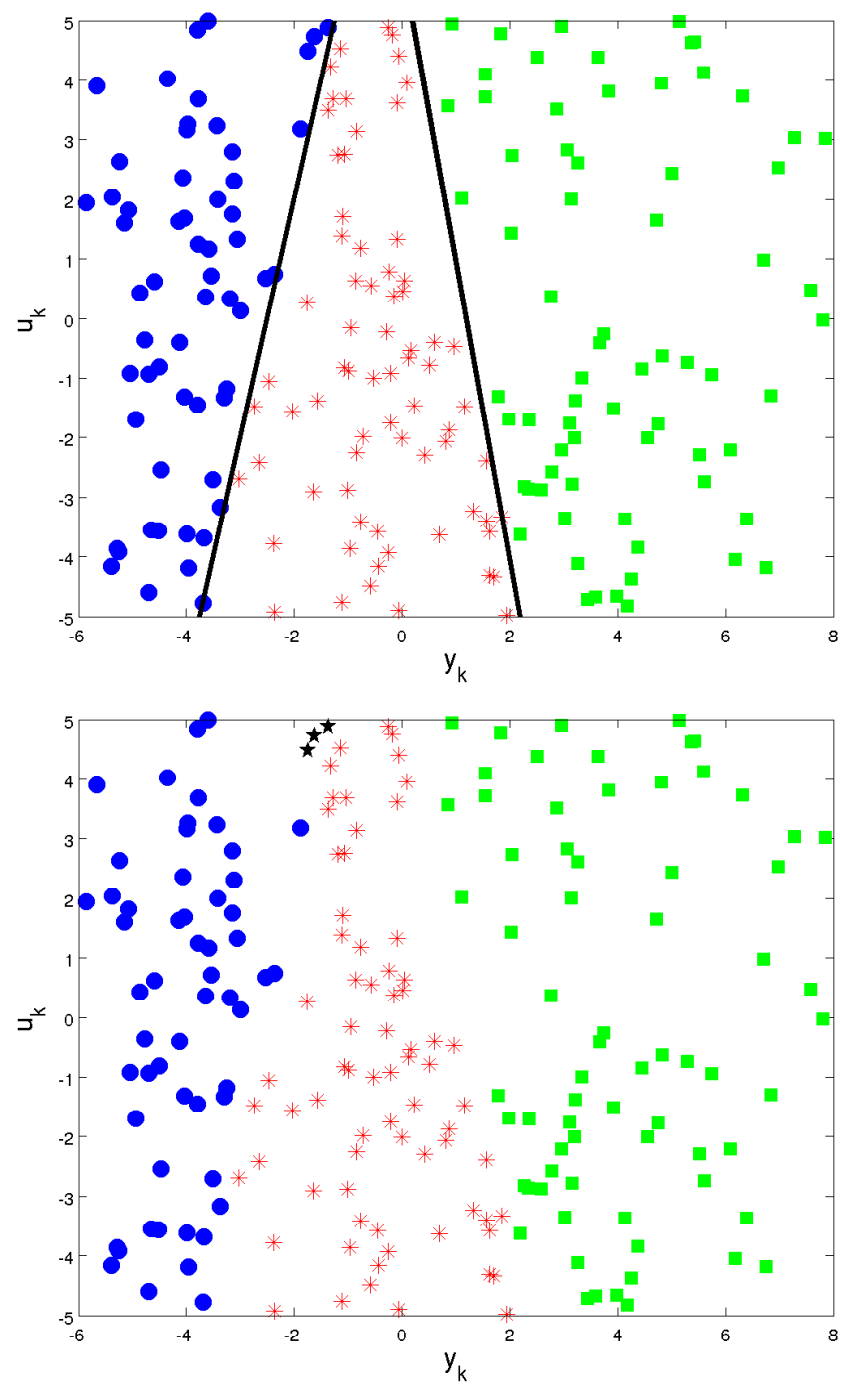

Fig. 3. Example 3.2. Top plot, generated data. Lines divide the three partitions. Bottom plot, color-coded estimates of $\theta$.

Example 3.3. Approximation of a Nonlinear Function

Consider

$$
y_{t}=f\left(u_{t}\right)+e_{t}, \quad f\left(u_{t}\right)=e^{-u_{t}}, e_{t} \sim N(0,0.001) .
$$

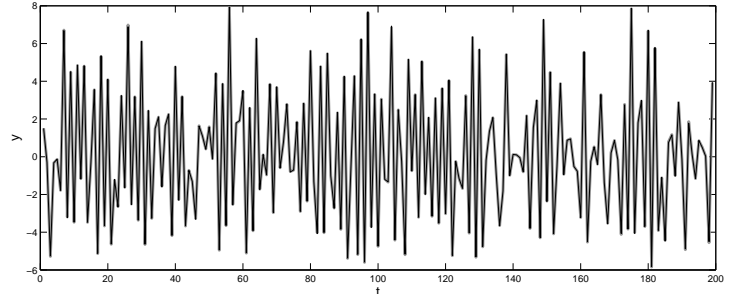

Fig. 4. Example 3.2. Noise-free $y$ (black thin) and estimated $y$ (thick gray line) for $k=1, \ldots, 200$.

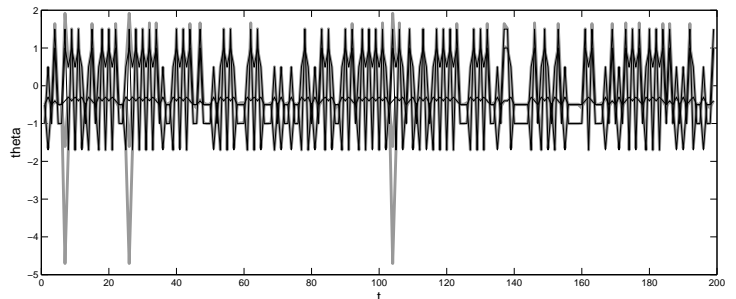

Fig. 5. Example 3.2. True $\theta$ (black thin) and estimated $\theta$ (thick gray line) for $k=1, \ldots, 200$.

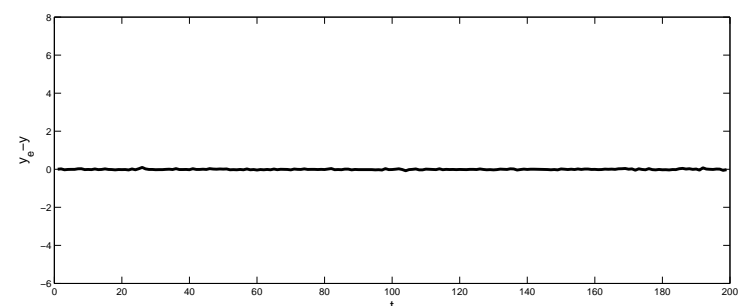

Fig. 6. Example 3.2. Difference between noise-free $y$ and estimated $y$ for $k=1, \ldots, 200$.

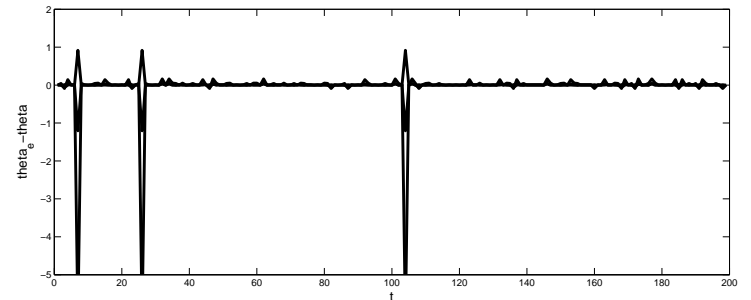

Fig. 7. Example 3.2. Difference between true $\theta$ and estimated $\theta$ for $k=1, \ldots, 200$.

Generate 100 observations by letting $u \sim U(0,5)$. The observations are shown in Figure 8. Let now use the proposed method to generate a piecewise affine approximation to $f\left(u_{t}\right)=e^{-u_{t}}$. $\lambda$ here controls the trade-off between the fit and the number of segments. $\lambda=0.01$ gives the result given in Figure 9 and $\lambda=0.05$ gives the result given in Figure 10. In both cases, the kernel defined by (9), $\Gamma=1$ and $p=1$ were used. 


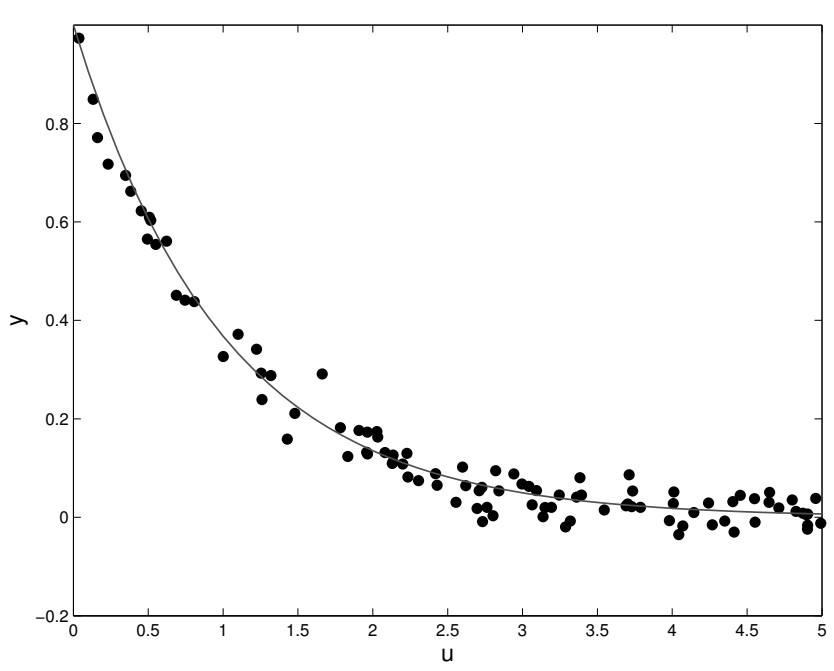

Fig. 8. Example 3.3. Observed $y$ :s and $f$ (thin gray line).

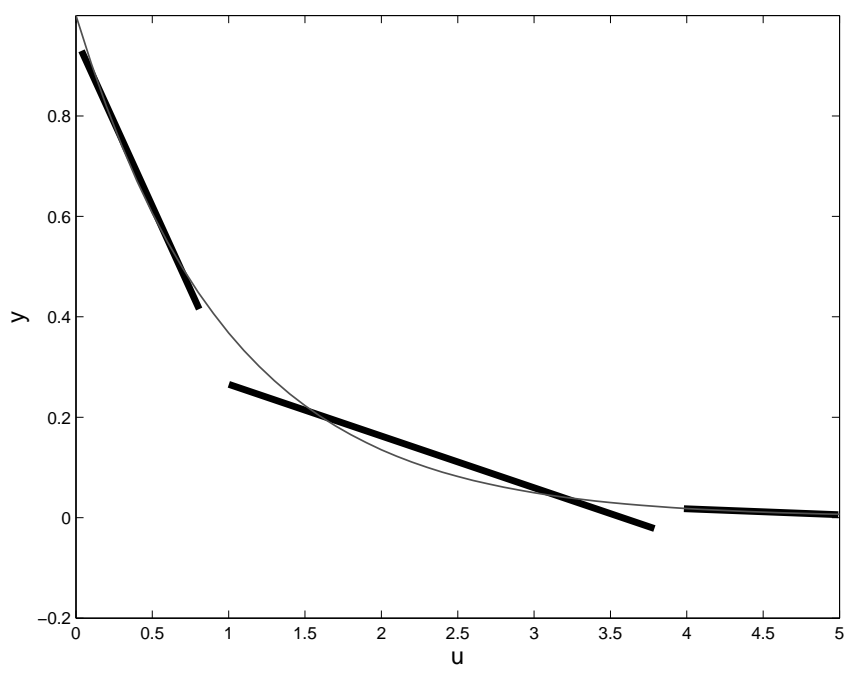

Fig. 9. Example 3.3. Approximated $f$ (thick black line) and $f$ (thin gray line). $\lambda=0.01$.

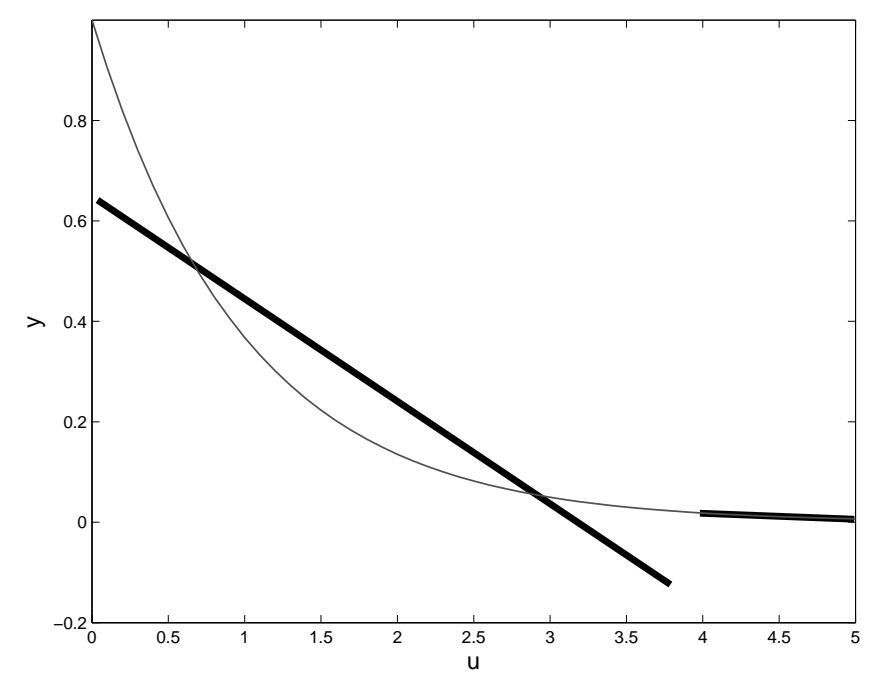

Fig. 10. Example 3.3. Approximated $f$ (thick black line) and $f$ (thin gray line). $\lambda=0.05$.

\section{CONCLUSION}

A method for piecewise affine system identification has been presented. The method builds on the assumption that the matrix composed of pairwise differences between the regressor parameter vectors associated with the observations is a sparse matrix. The formulation takes the shape of a least-squares problem with sum-of-norms regularization over regressor parameter differences, a generalization of $\ell_{1}$ regularization. The regularization constant is used to trade off fit and the number of partitions. Numerical illustrations on previously known examples from the literature shows that the proposed method performs well in comparison to know PW affine systems identification methods.

There are several interesting extensions of proposed scheme. For example, a piecewise nonlinear function could be estimated by applying a regularization as in (8) to Support Vector Regression (SVR, see e.g., Suykens and Vandewalle [1999]).

\section{REFERENCES}

Alberto Bemporad, Andrea Garulli, Simone Paoletti, and Antonio Vicino. A greedy approach to identification of piecewise affine models. In Proceedings of the 6th international conference on Hybrid systems (HSCC'03), pages 97-112, Prague, Czech Republic, 2003. SpringerVerlag.

Alberto Bemporad, Andrea Garulli, Simone Paoletti, and Antonio Vicino. A bounded-error approach to piecewise affine system identification. IEEE Transactions on Automatic Control, 50(10):1567-1580, October 2005.

Emmanuel J. Candès, Justin Romberg, and Tao Terence. Robust uncertainty principles: exact signal reconstruction from highly incomplete frequency information. IEEE Transactions on Information Theory, 52:489-509, February 2006.

Emmanuel J. Candès, Michael B. Wakin, and Stephen Boyd. Enhancing sparsity by reweighted $\ell_{1}$ minimization. Journal of Fourier Analysis and Applications, special issue on sparsity, 14(5):877-905, December 2008.

David L. Donoho. Compressed sensing. IEEE Transactions on Information Theory, 52(4):1289-1306, April 2006.

Giancarlo Ferrari-Trecate, Marco Muselli, Diego Liberati, and Manfred Morari. A clustering technique for the identification of piecewise affine systems. Automatica, $39(2): 205-217,2003$.

Michael Grant and Stephen Boyd. Graph implementations for nonsmooth convex programs. In Vincent D. Blondel, Stephen Boyd, and Hidenori Kimura, editors, Recent Advances in Learning and Control, Lecture Notes in Control and Information Sciences, pages 95-110. Springer-Verlag Limited, 2008. http://stanford.edu/ $\sim$ boyd/graph_dcp.html.

Michael Grant and Stephen Boyd. CVX: Matlab software for disciplined convex programming, version 1.21. http: //cvxr.com/cvx, August 2010.

Seung-Jean Kim, Kwangmoo Koh, Michael Lustig, Stephen Boyd, and Dimitry Gorinevsky. An interiorpoint method for large-scale 11-regularized least squares. IEEE Journal of Selected Topics in Signal Processing, 1 (4):606-617, December 2007. 
Seung-Jean Kim, Kwangmoo Koh, Stephen Boyd, and Dimitry Gorinevsky. $\ell_{1}$ trend filtering. SIAM Review, 51(2):339-360, 2009.

Johan Löfberg. Yalmip : A toolbox for modeling and optimization in MATLAB. In Proceedings of the CACSD Conference, Taipei, Taiwan, 2004. URL http: //control.ee.ethz.ch/ joloef/yalmip.php.

Hayato Nakada, Kiyotsugu Takaba, and Tohru Katayama. Identification of piecewise affine systems based on statistical clustering technique. Automatica, 41(5):905-913, 2005.

Henrik Ohlsson, Fredrik Gustafsson, Lennart Ljung, and Stephen Boyd. State smoothing by sum-of-norms regularization. In Proceedings of the 49th IEEE Conference on Decision and Control, Atlanta, USA, December 2010a.

Henrik Ohlsson, Fredrik Gustafsson, Lennart Ljung, and Stephen Boyd. Trajectory generation using sum-ofnorms regularization. In Proceedings of the 49th IEEE Conference on Decision and Control, Atlanta, USA, December 2010b.

Henrik Ohlsson, Lennart Ljung, and Stephen Boyd. Segmentation of ARX-models using sum-of-norms regularization. Automatica, 46(6):1107-1111, 2010c.

Necmiye Ozay, Mario Sznaier, Constantino M. Lagoa, and Octavia Camps. A sparsification approach to set membership identification of a class of affine hybrid systems. In Proceedings of the 47th IEEE Conference on Decision and Control, pages 123-130, December 2008.

Jacob Roll. Piecwise linear solution paths with application to direct weight optimizatiom. Automatica, 44:27452753, 2008.

Jacob Roll, Alberto Bemporad, and Lennart Ljung. Identification of piecewise affine systems via mixed-integer programming. Automatica, 40(1):37-50, 2004.

Johan A. K. Suykens and Joos Vandewalle. Least squares support vector machine classifiers. Neural Processing Letters, 9(3):293-300, 1999.

Robert Tibsharani. Regression shrinkage and selection via the lasso. Journal of Royal Statistical Society $B$ (Methodological), 58(1):267-288, 1996.

Vladimir Vapnik. The Nature of Statistical Learning Theory. Springer, New York, 1995.

René Vidal, Stefano Soatto, Yi Ma, and Shankar Sastry. An algebraic geometric approach to the identification of a class of linear hybrid systems. In Proceedings of the 42nd IEEE Conference on Decision and Control, volume 1, pages 167-172, December 2003.

Ming Yuan and Yi Lin. Model selection and estimation in regression with grouped variables. Journal of the Royal Statistical Society, Series B, 68:49-67, 2006. 